\title{
Fine mapping of a candidate gene for cool- temperature-induced albinism in ornamental kale
}

\author{
Chenghuan Yan ${ }^{1,2}$, Liying Peng ${ }^{1}$, Lei Zhang ${ }^{1}$ and Zhengming Qiu $^{2^{*}}$ (D)
}

\begin{abstract}
Background: The symptoms of cool-temperature-induced chlorosis (CTIC) are widely existed in higher plants. Although many studies have shown that the genetic mechanism of CTIC is generally controlled by recessive genes in model plants, the dominant inheritance of albinism has not been reported thus far. Here, two CTIC mutants, Red Kamome and White Kamome, were utilized to analyse the inheritance of the albino trait in ornamental kale. The objective of this investigation is to fine-map the target locus and identify the most likely candidate genes for albinism.

Results: Genetic analysis revealed that the albinism in the inner leaves of ornamental kale followed semi-dominant inheritance and was controlled by a single locus in two segregating populations. BSR-seq in combination with linkage analysis was employed to fine-map the causal gene, named AK (Albino Kale), to an approximate $60 \mathrm{~kb}$ interval on chromosome C03. Transcriptome data from two extreme pools indicated that the differentially expressed gene of Bol015404, which encodes a cytochrome P450 protein, was the candidate gene. The Bol015404 gene was demonstrated to be upregulated in the albino leaves of ornamental kale by qPCR. Additionally, the critical temperature for the albinism was determined between $10^{\circ} \mathrm{C}$ and $16^{\circ} \mathrm{C}$ by gradient test.

Conclusions: Using two independent segregating populations, the albino mutants were shown to be controlled by one semi-dominant gene, $A K$, in ornamental kale. The Bol015404 gene was co-segregated with albinism phenotypes, suggesting this unknown function P450 gene as the most likely candidate gene. The albino trait appeared caused by the low temperatures rather than photoperiod. Our results lay a solid foundation on the genetic control of albinism in ornamental kale.
\end{abstract}

Keywords: Albino trait, Semi-dominant inheritance, BSR-seq, Cytochrome P450 gene, Ornamental kale

\section{Background}

Chlorophyll biosynthesis is the most important biochemical process on our planet [1]. The chlorophyll biosynthetic pathway occurs in chloroplasts, and involves many enzyme-catalysed reactions [2]. Therefore, chloroplasts are unique units of photosynthesis in green plants that generate multiple metabolic products of the

\footnotetext{
* Correspondence: qiusunmoon@163.com

${ }^{2}$ Hubei Key Laboratory of Vegetable Germplasm Enhancement and Genetic Improvement, Institute of Economic Crops, Hubei Academy of Agricultural Sciences, Wuhan 430064, People's Republic of China

Full list of author information is available at the end of the article
}

photosynthetic processes [3]. Numerous studies have reported that chloroplasts are derived from proplastids, and the formation of chloroplasts involves the regulation of plastid and nuclear genes [4]. To date, substantial evidences have revealed that the biogenesis of chloroplasts is precisely regulated by a series of genes.

Low temperature is a common abiotic stress for most Brassica plants and is necessary for vernalization and reproduction. The symptoms of cool-temperatureinduced chlorosis (CTIC) are usually observed in higher plants, such as Arabidopsis [5], rice [6, 7], maize [8], etc. Using genetic analysis approaches, many temperature-

(c) The Author(s). 2020 Open Access This article is licensed under a Creative Commons Attribution 4.0 International License, which permits use, sharing, adaptation, distribution and reproduction in any medium or format, as long as you give appropriate credit to the original author(s) and the source, provide a link to the Creative Commons licence, and indicate if changes were made. The images or other third party material in this article are included in the article's Creative Commons licence, unless indicated otherwise in a credit line to the material. If material is not included in the article's Creative Commons licence and your intended use is not permitted by statutory regulation or exceeds the permitted use, you will need to obtain permission directly from the copyright holder. To view a copy of this licence, visit http://creativecommons.org/licenses/by/4.0/. The Creative Commons Public Domain Dedication waiver (http://creativecommons.org/publicdomain/zero/1.0/) applies to the data made available in this article, unless otherwise stated in a credit line to the data. 
sensitive mutants have been identified in rice, including tsc-1 [9], cde1 [10], ysa [11], tcd5 [12], etc. In mung beans, etiolated seedlings in the dark were completely repressed at $10{ }^{\circ} \mathrm{C}$ and were unable to turn green again under normal light conditions [13]. Thus, both dicots and monocots have CTIC symptoms, which are universal phenomena in higher plants. However, the molecular mechanisms of cool-temperature-induced chloroplast deficiency have not been fully elucidated.

Ornamental kale (Brassica oleracea var. acephala) and its related varieties, including curly kale $(B$. oleracea var. sabellica L.), thousand-head kale (B. oleracea var. ramosa DC.), marrow-stem kale (B. oleracea var. medullosa Thellg.), etc., belong to the members of kale of B. oleracea [14]. For human purposes, kales are divided into two main types: edible kale, which is used as a vegetable or fodder crop (such as curly kale), and ornamental kale, which is used in landscaping. Ornamental kale is one of the most popular ornamental crop worldwide. In China, ornamental kale is generally used as a landscape plant in winter due to its colourful morphology. The main colours of the cultivated ornamental kales are white and red. The investigation of CTIC symptoms in kale can be traced back to the middle of the twentieth century. In 1959, Martin discovered a dominant gene that likely controlled the albinism in winter, but a contradictory conclusion was made in summer [15]. Another study showed that the albino trait in ornamental kale is related to a chlorophyll deficiency in the inner leaves [16]. In a previous study, a red kale called Red Kamome was discovered to retain two independent traits for both anthocyanin accumulation and albinism, revealing that the albino phenotypes may be controlled by a different locus [17]. However, the genetic and molecular mechanisms of albinism remain poorly understanding in kale.

Albinism is a unique variation in kale that can be produced by undiscovered and infrequent genetic mechanisms. To confirm this hypothesis, the inheritance of this trait was carefully analysed using two independent segregating populations. This research aimed to elucidate this genetic relationship for further identification of the candidate gene. In this study, a rare semi-dominant inheritance pattern was repeatedly identified for albinism in ornamental kale. Furthermore, the target trait was fine-mapped within a narrow interval using BSR-seq and linkage analysis. Our study sheds light on the genetic mechanism controlling albinism in kale, and provides useful information for the further functional characterization of the candidate gene.

\section{Results}

Genetic analysis of the albino trait in ornamental kale

Two albino mutants, RK01 and WK02, were utilized to analyse the inheritance of albinism in ornamental kale. A $\mathrm{F}_{2}$ segregating population was generated by WK02
(Fig. 1a) with albino phenotype in the inner leaves and CK04 (Fig. 1b) with green leaves, which $F_{1}$ progenies showed slight albino phenotype in the inner leaves (Fig. $1 c)$. The $F_{2}$ progenies exhibited three leaf colour phenotypes, including 48 albino plants, 92 slight albino plants and 38 normal plants, with a segregation ratio of 1:2:1 $\left(\chi^{2}=1.33, P=0.52>0.05\right.$, Fig. $\left.1 \mathrm{~d}\right)$. The result indicates that the albino trait is controlled by a semi-dominant locus, named Albino Kale (AK), in WK02. Additionally, the albino phenotype was also discovered in the $F_{1}$ plants and $\mathrm{BC}_{1}$ population of $\mathrm{RK} 01$ and green cabbage [17]. A total of $603 \mathrm{BC}_{1}$ progenies were used to analyse the inheritance of the albinism independently. Phenotyping of 603 progenies revealed that the segregation ratio of 304 albino individuals and 299 green individuals were 1:1 $\left(\chi^{2}=0.04, P=0.84>0.05\right.$, Figure S1 and Table 1$)$. Furthermore, a small $\mathrm{BC}_{1} \mathrm{~F}_{2}$ population was selected to further determine the genetic relationship. Progenies of the $\mathrm{BC}_{1} \mathrm{~F}_{2}$ population showed three leaf colour phenotypes, including 27 albino plants, 52 slight albino plants and 20 normal plants, with Mendelian ratio of 1:2:1 $\left(\chi^{2}=1.24, P=0.54>0.05\right.$, Figure S2 and Table 1$)$. These results also indicate that the albinism of ornamental kale is a unique semi-dominant trait, which is probably controlled by single gene. Chlorophyll contents of parental lines were measured at four-month-old stage. Chlorophyll contents of the albino region in RK01 and WK02 were significantly lower than that of the inner leaves in green cabbage and CK04 $(P<0.001)$, while it had no significant difference in the outer leaves among RK01, WK02, CK04 and green cabbage (Fig. 1e). The findings suggested that the albino phenotype might be caused by repressing the chlorophyll biosynthesis and/or chloroplast development in ornamental kale.

\section{Phenotyping and chloroplast analysis in albino kale genotypes}

To understand how chlorophyll and/or chloroplast cause albino phenotypes, transmission electron microscopy was ultilized to observe the ultrastructures of the chloroplasts in WK02 and green cabbage. Three regions in the leaves of WK02, including all-green, albino-green and all-albino tissues, were selected for the observation of their chloroplast morphology (Fig. 2b), and green cabbage was used as a control (Fig. 2a). Unlike in green cabbage, the number of chloroplasts gradually decreased to zero from the all-green to the all-albino tissues in WK02 (Fig. 2c-f). In the all-green tissues of WK02, the chloroplast morphology showed normal development at low temperature (Fig. 2h). In addition, the chloroplast ultrastructure revealed a loose arrangement and abnormal grana stacks, and some vacuoles were even detected in the albino-green tissues (Fig. 2i). Interestingly, chloroplasts were not observed in the all-albino tissues (Fig. 


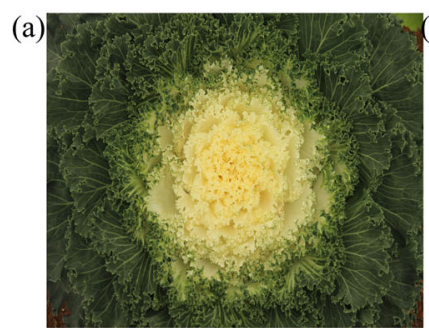

WK02(P1)

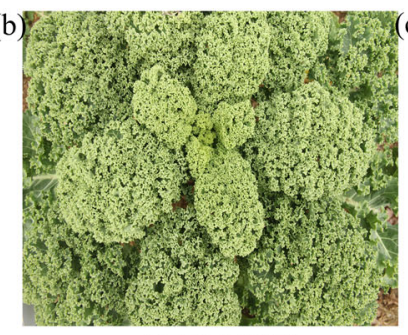

CK04(P2)

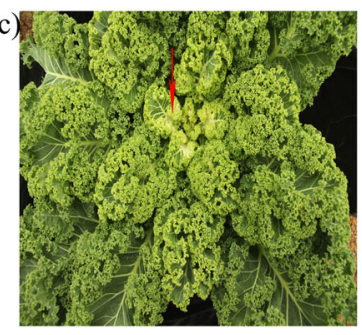

$\mathrm{F}_{1}$ (d)

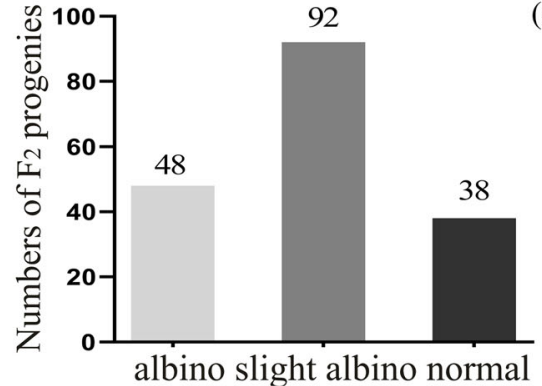

(e)

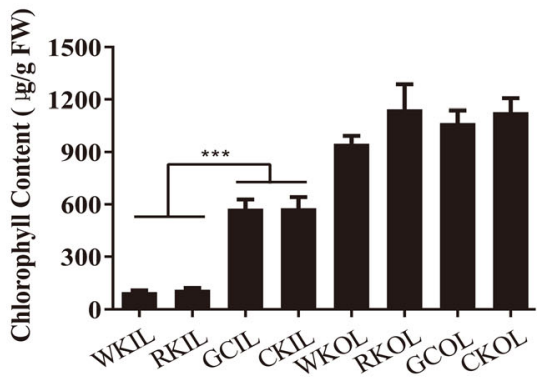

Fig. 1 The phenotypes and chlorophyll contents of the parental lines and their progenies. a WK02. b CK04 (wild type). c The phenotype of $F_{1}$ progeny. $\mathbf{d}$ The phenotypic distribution of $\mathrm{F}_{2}$ progenies. e Chlorophyll contents in WK02, RK01, green cabbage and CK04. RK, RK01; GC, green cabbage; WK, WK02; CK, CK04; OL, outer leaves; IL, inner leaves. *** means $p<0.001$ by student's $t$ test

2f), but mitochondria were (Fig. 2j). These results were consistent with the phenotype and chlorophyll content in WK02. Therefore, we inferred that no chloroplasts were transformed from proplastids in the all-albino tissues of WK02. To further confirm this hypothesis, protoplasts of WK02 and green cabbage were isolated and observed. Similar to those of the green cabbage, the protoplasts of all-green tissues had normal chloroplast morphology in WK02 (Fig. 2k-l), and no chloroplasts were identified in the protoplasts of all-albino tissues (Fig. 2n). In the green-albino tissues, the morphology of both normal chloroplast and chloroplast-free was clearly observed, revealing an intermediate state between the all-green and all-albino tissues (Fig. 2m). These results indicate that the albino phenotype in the inner leaves of White Kamome was caused by chloroplast deficiency.

\section{AK gene mapping}

BSR-seq was employed to preliminarily map the $A K$ gene. Two pools, A-pool and N-pool, generated $5.29 \mathrm{~Gb}$ and $6.21 \mathrm{~Gb}$ of clean data by RNA-seq, respectively. The $\Delta$ (SNP-index) graph was calculated based on the data of N-pool and A-pool against the reference genome 0212 [18]. A peak for $A K$ locus occurred in the front of chromosome C03 (Fig. 3a), indicating a single locus controlling the albinism in ornamental kale.

At $C I$ values of 95 and $99 \%$, the $A K$ gene was anchored in $0-15.10(P<0.05) \mathrm{Mb}$ and $0-13.30 \mathrm{Mb}(P<0.01)$ on chromosome C03 (Fig. 3a). Furthermore, we screened 93 randomly selected progenies of the $\mathrm{BC}_{1}$ population using six molecular markers, including BoY001, BoY003, BoY006, BoY007, BoY008 and BoY009. Using the 93 progenies, the $A K$ gene was preliminary mapped between molecular markers BoY001 (300, $692 \mathrm{bp})$ and BoY003 (1, 229, 918 bp) (Fig. 3b).

To fine-map the $A K$ gene, we screened 603 progenies of the $\mathrm{BC}_{1}$ population using molecular markers BoY001 and BoY003, with identifying twenty and twenty-two recombinants, respectively. New polymorphic markers were developed between BoY001 and BoY003, including BoY002, BoY010, BoY011, BoY012, BoY013 and BoY015. Finally, $A K$ gene was delimited in the region between molecular markers BoY010 (754, $756 \mathrm{bp})$ and BoY011 (815, $202 \mathrm{bp})$, with a physical region of approximate $60 \mathrm{~kb}$ and genetic distance of $0.33 \mathrm{cM}$ (Fig. 3c).

Table 1 The Chi-square $\left(x^{2}\right)$ of leaf colour segregation in the $\mathrm{BC}_{1}$ and its derivative populations

\begin{tabular}{lllllll}
\hline Population & Plant numbers & No. albino individuals & No. slight albino individuals & No. normal individuals & Expected ratio & $X^{2}$ \\
\hline$F_{1}$ & 21 & - & 21 & 0 & - & - \\
$B_{1}$ & 603 & - & 304 & 299 & $1: 1$ & $0.04^{a}$ \\
$B_{1} C_{1}$ & 99 & 27 & 52 & 20 & $1: 2: 1$ & $1.24^{a}$ \\
\hline
\end{tabular}

${ }^{a} X^{2}>X_{0.05}^{2}=3.84$ were regarded as significant difference 
(a)

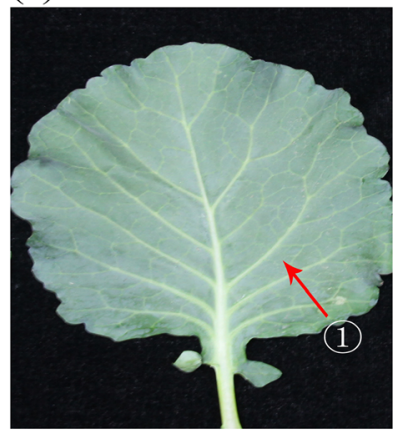

(1)
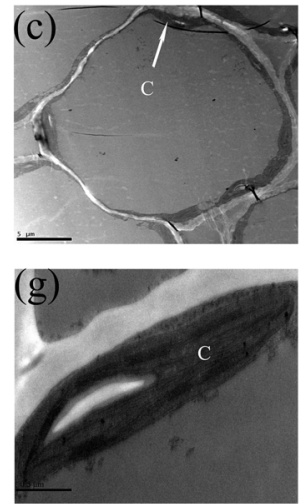

(k)

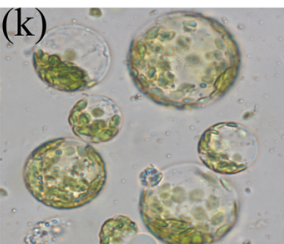

(2)
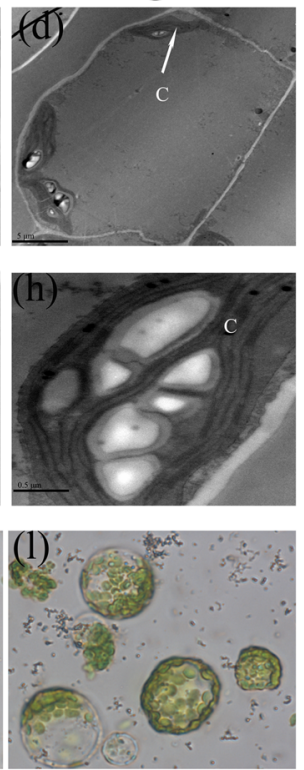

(b)

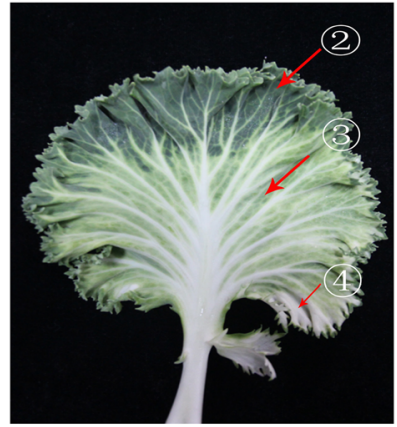

(3)
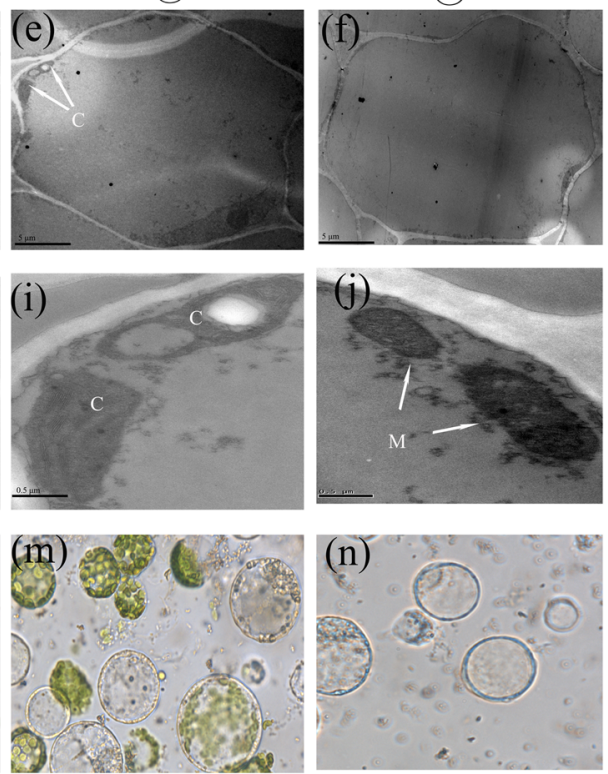

Fig. $\mathbf{2}$ Cytologic characteristics of green cabbage and WKO2. a Leaf of green cabbage. $\mathbf{b}$ Leaf of WK02. Transmission electron microscope images of position (1) shown in $\mathbf{c}$ and $\mathbf{g}$, position (2) shown in $\mathbf{d}$ and $\mathbf{h}$, position (3) shown in $\mathbf{e}$ and $\mathbf{i}$, position (4) shown in $\mathbf{f}$ and $\mathbf{j}$. Protoplast images (60x) of position (1)-(4) shown in $\mathbf{k}-\mathbf{n}$, respectively. The bars of $\mathbf{c}$-f represent $5 \mu \mathrm{m}$; The bars of $\mathbf{g}$-j represent $0.5 \mu \mathrm{m}$. C, chloroplast; $\mathrm{M}$, mitochondria

\section{Candidate $A K$ gene prediction}

In the target region, thirteen predicted genes were obtained between BoY010 and BoY011 (Table 2, Fig. 3d). Then, FGENESH and GENESCAN were operated to identify thirteen ORFs, which were consistent with the reference genome. Five predicted genes, including Bol015395, Bolo15396, Bol015399, Bol015401 and Bol015402, encode uncharacterized proteins. Bol015394 is likely to encode a clathrin adaptor complex subunit protein that shares $78.1 \%$ amino acid identity with AT5G05010. Bol015397 encodes ubiquitin-conjugating enzyme 22, which is involved in female gametophyte development [19]. Bol015398 is a MYBC1-like transcription factor that negatively regulates the freezing tolerance in Arabidopsis [20]. Bol015406 encodes a cellulose synthase involved in the cellulose biosynthesis process [21]. Bol015400, Bol015403 and Bol015405 are related to fundamental biological processes, such as repressing cysteine proteinase, RNA polymerase transcription, and autophagosome assembly, respectively. Bol015404 encodes a cytochrome P450 708A subfamily protein, which is orthologous with $A T 3 G 44970$, sharing $73.7 \%$ amino acid identity. The function of Bol015404 has not yet been verified. In the fine-mapping region, only one gene of Bol015404 was differentially expressed between the A-pool and N-pool (FPKM value of the A-pool/N-pool $\approx 27.1$ ). Thus, the Bol015404 gene was temporarily considered a candidate gene (Fig. $3 \mathrm{~d}$ ).

To determine the possible candidate gene, the expression levels of Bol015404 under different temperature treatments were detected by qPCR. The results revealed that the expression levels of Bol015404 were relatively low in the root and stem tissues of WK02 and green cabbage (Fig. 4). However, Bol015404 had higher expression in the inner leaves of WK02 than in those of green cabbage $(P<0.001)$ at $10^{\circ} \mathrm{C}$ and $24^{\circ} \mathrm{C}$. Compared with 

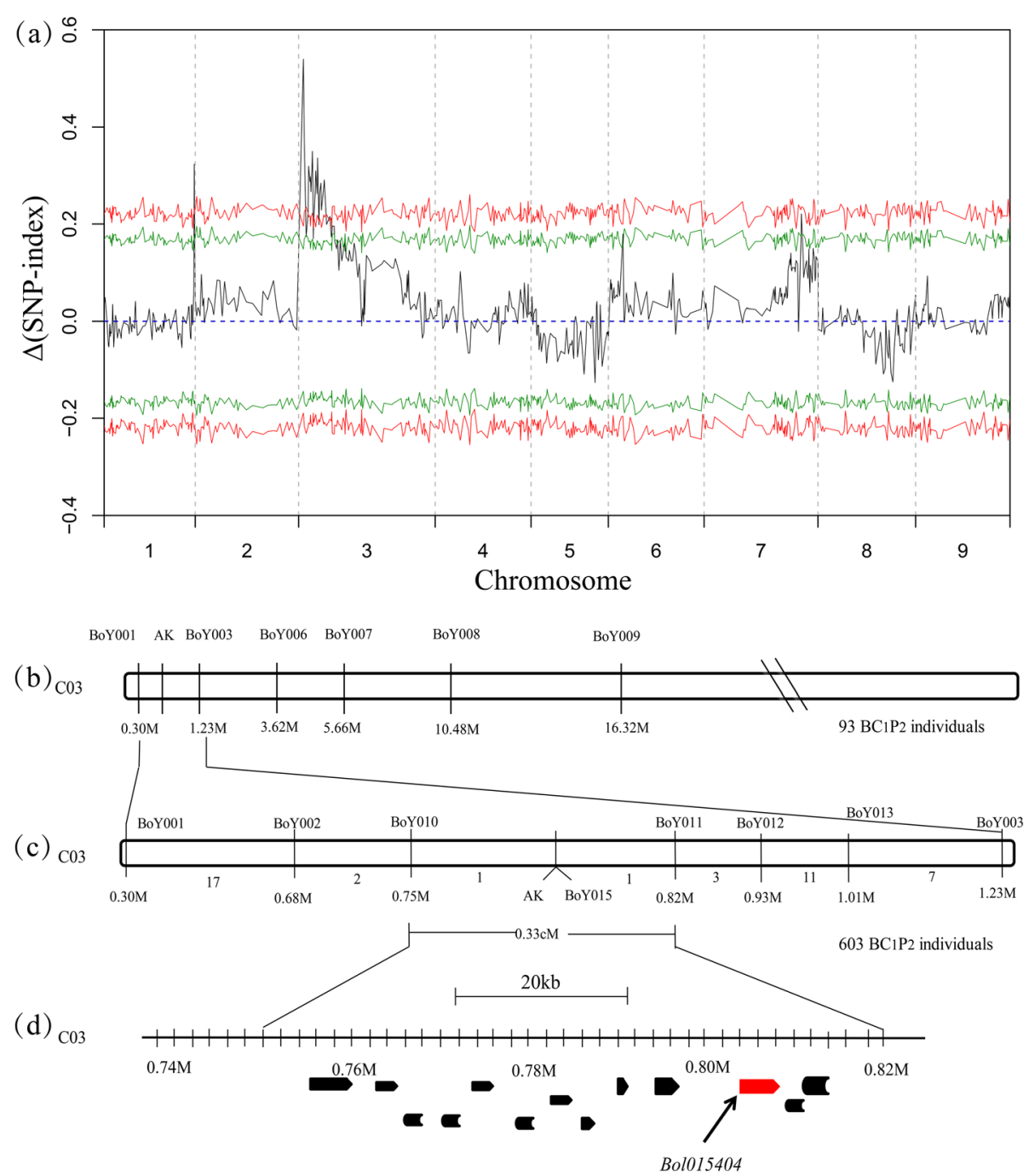

Fig. 3 Mapping of the $A K$ gene in the $\mathrm{BC}_{1}$ population a The average $\Delta$ (SNP-index) graph based on the data of N-pool and A-pool against reference genome 02-12 (X-axis). Peak of target region was shown on Chromosome C03. The Cls were revealed with green lines $(P<0.05)$ and red lines $(P<0.01)$. b The AK gene was mapped between molecular markers BoY001 and BoY003 using 93 randomly selected $\mathrm{BC}_{1}$ individuals. $\mathbf{c}$ Fine mapping the AK gene between molecular markers BoY010 (754, 756 bp) and BoY011 (815, 202 bp). The numbers below chromosome indicate the number of recombinants between two markers. $\mathbf{d}$ Schematic diagram of predicted genes in the AK locus. The broad arrows represent predicted thirteen genes in the candidate region. The Bol015404 was considered a candidate gene

those in the $24^{\circ} \mathrm{C}$ treatment, the expression levels of Bol015404 were significantly upregulated in the inner leaves of WK02 at $10^{\circ} \mathrm{C}(P<0.01)$. The findings indicate that the expression of Bol015404 in the inner leaves may be induced by low temperature. Furthermore, a SNP marker, BoY015, located in the intron region of Bol015404, co-segregated with the phenotypes in the $\mathrm{BC}_{1}$ population (Fig. 3c). Thus, Bol015404 was regarded as the most likely candidate gene regulating albinism.

\section{Sequence analysis of Bol015404}

We amplified and sequenced the genomic sequences (including approximate $2.5 \mathrm{~kb}$ promoter region) of Bol015404 in RK01, WK02 and green cabbage. The genomic sequences revealed complete identity in the intron and promoter regions between RK01 and WK02 (Text S1). In the coding regions, two albino kales, RK01 and WK02, and green cabbage had $100 \%$ sequence identity (Figure S3). Interestingly, the splice site of intron 6 was occasionally GC-AG rather than GT-AG in the three parental lines. We analysed the promoter sequences of WK02 and green cabbage in detail. Unlike in green cabbage, 12 point mutations were distributed in the promoter region of $\mathrm{WK} 02$, and a $10 \mathrm{bp}$ deletion was discovered at the -2066 bp location (Fig. 5). We inferred that these mutations in the promoter region might be responsible for the induced expression of Bol015404 in the inner leaves of WK02. 
Table 2 The thirteen predicted genes between molecular markers BoY010 and BoY011

\begin{tabular}{lllll}
\hline Gene name & start & stop & Length (bp) & identifier \\
Bol015394 & 756,300 & 763,338 & 2013 & Clathrin adaptor complexes subunit protein \\
Bol015395 & 763,580 & 764,449 & 204 & Uncharacterized protein \\
Bol015396 & 765,035 & 765,273 & 153 & Uncharacterized protein \\
Bol015397 & 767,150 & 768,367 & 618 & Ubiqutin-conjugating enzyme E2 22-like \\
Bol015398 & 775,158 & 775,907 & 750 & Transcription factor MYBC1-like \\
Bol015399 & 778,895 & 780,322 & 951 & Uncharacterized protein \\
Bol015400 & 780,861 & 781,878 & 687 & Cysteine proteinase inhibitor 7 \\
Bol015401 & 782,878 & 783,426 & 549 & Uncharacterized protein \\
Bol015402 & 791,186 & 791,626 & 441 & Uncharacterized protein \\
Bol015403 & 795,555 & 797,410 & 1191 & Probable mediator of RNA polymerase II transcription subunit 26b \\
Bol015404 & $\mathbf{8 0 5 , 0 5 5}$ & $\mathbf{8 0 8 , 1 0 2}$ & $\mathbf{1 3 7 7}$ & Cytochrome P450 708A family protein \\
Bol015405 & 808,399 & 809,967 & 1119 & Autophagy-related protein 18E \\
Bol015406 & 810,685 & 813,318 & 1884 & Cellulose synthase A catalytic subunit 3 \\
\hline
\end{tabular}

\section{Albinism induced by low temperature rather than photoperiod}

The albino phenotype of ornamental kale was usually observed in autumn and winter fields. We speculated that the albinism was induced by low temperature and/or a short photoperiod. To determine the environmental factors, four temperature and photoperiod treatmeats were performed to explore the key abiotic factor. The LD and SD photoperiods did not cause significant differences in chlorophyll contents between WK02 and green cabbage. However, the phenotype of WK02 revealed obvious albinism in the inner leaves, and its chlorophyll content was significantly lower under the low temperature $\left(10^{\circ} \mathrm{C}\right)$ than under the normal temperature $\left(24^{\circ} \mathrm{C}\right)$ (Fig. 6a). Thus, the albino phenotype was induced by low temperature rather than by the photoperiod in ornamental kale.
To explore the critical temperature for albinism, a temperature gradient test was performed in this study. The results showed that the inner leaves of WK02 gradually developed the albinism at $4{ }^{\circ} \mathrm{C}$ and $10{ }^{\circ} \mathrm{C}$, but it always exhibited a normal green colour in the inner leaves after three weeks at $16^{\circ} \mathrm{C}$ and $24^{\circ} \mathrm{C}$ (Fig. 6c). Therefore, the chlorophyll content of WK02 decreased significantly under low temperatures $\left(4{ }^{\circ} \mathrm{C}\right.$ and $\left.10{ }^{\circ} \mathrm{C}\right)$ compared with other temperatures $(P<0.001$, Fig. $6 \mathrm{~b})$.

\section{Discussion}

In this study, bulked segregant analysis (BSA) in combination with RNA-seq (BSR-seq) [18] was employed to preliminarily map the target region of the albino phenotype in ornamental kale. A single peak was identified on chromosome C03 (Fig. 3a) by calculating the values of $\Delta$
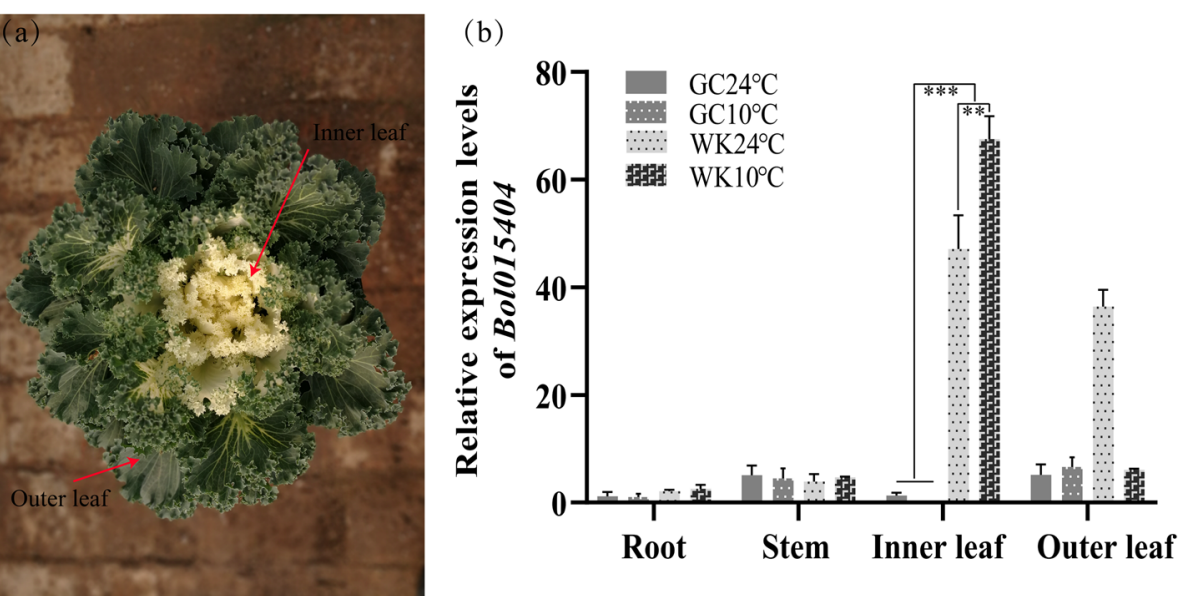

Fig. 4 Relative expression levels of Bol015404 (a) Phenotype of WK02 under low temperature. b Expression levels of Bol015404 in different tissues at $10^{\circ} \mathrm{C}$ and $24^{\circ} \mathrm{C}$. Data represent mean \pm SD $(n=3)$. The expression level of Bol015404 in the green cabbage at $24{ }^{\circ} \mathrm{C}$ was set as 1 . WK, WK02; GC, green cabbage. ${ }^{* *}$ and ${ }^{* * *}$ means $P<0.01$ and $P<0.001$ by student's $t$ test 


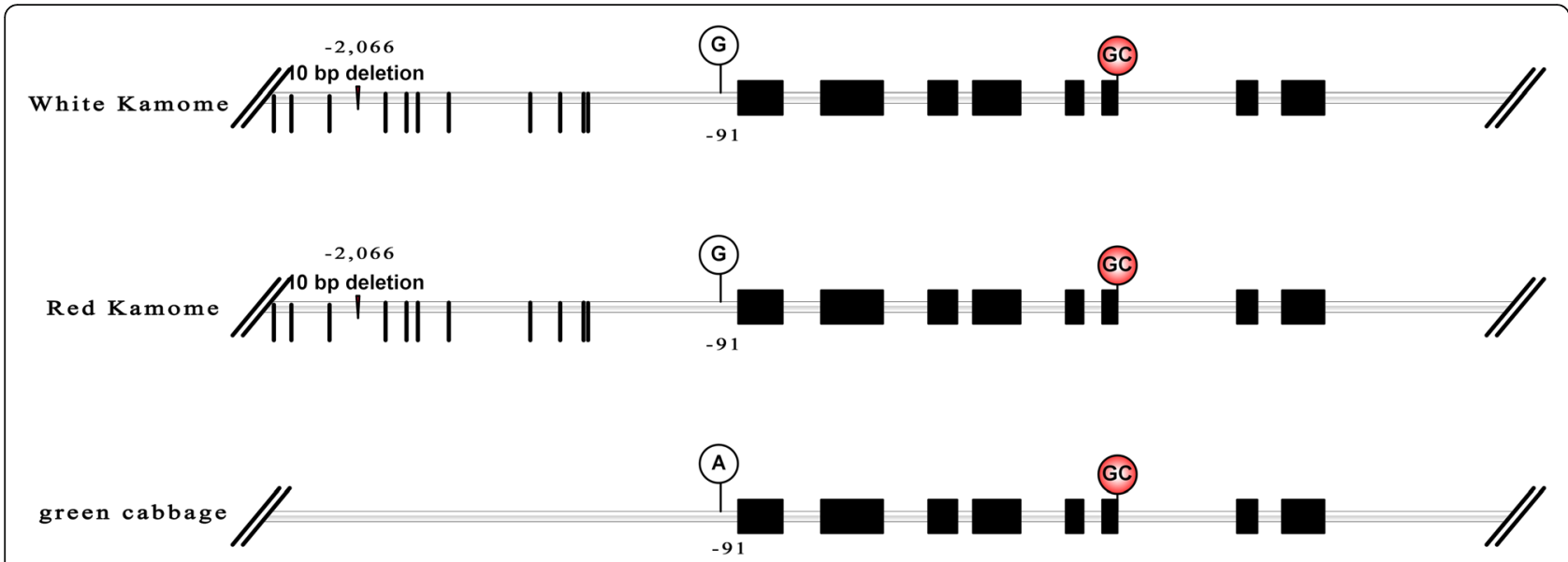

Fig. 5 Schematic diagram of Bol015404 alleles in WK02, RK01 and green cabbage

(SNP-index) between two pools, the A-pool and the Npool. These results demonstrated that BSR-seq was a powerful approach for identifying the corresponding region of the target trait. Additionally, BSA in combination with DNA resequencing (QTL-seq) can also promote the efficiency of genetic mapping [19]. Therefore, BSRseq and QTL-seq have been widely used to identify several causal genes in B. oleracea species, such as BoLl [20,
21], BoPs [22], BoMYB2 [17], BoCCD4 [23, 24]. Unlike QTL-seq, BSR-seq takes advantage of differentially expressed genes from two pools, which simultaneously provides more information for candidate gene selection. Additionally, we mapped the albino trait using the $\mathrm{BC}_{1}$ segregating population (Table S1), which the population had been utilized to elucidate the genetic mechanism of anthocyanin accumulation in ornamental kale [17].
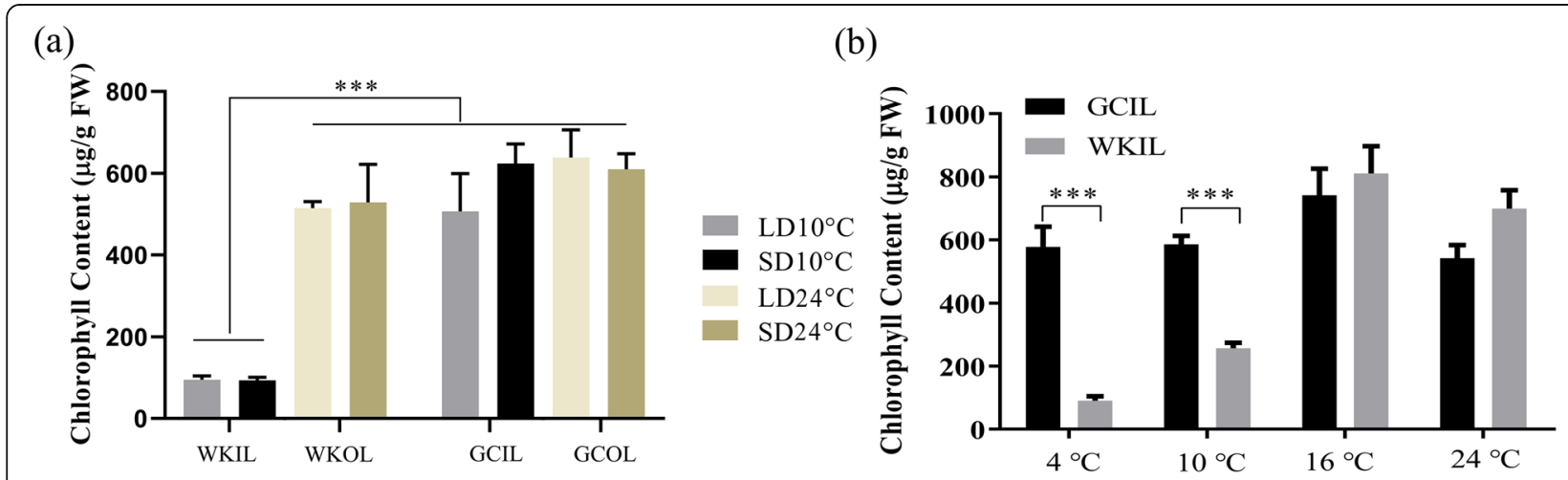

(c)
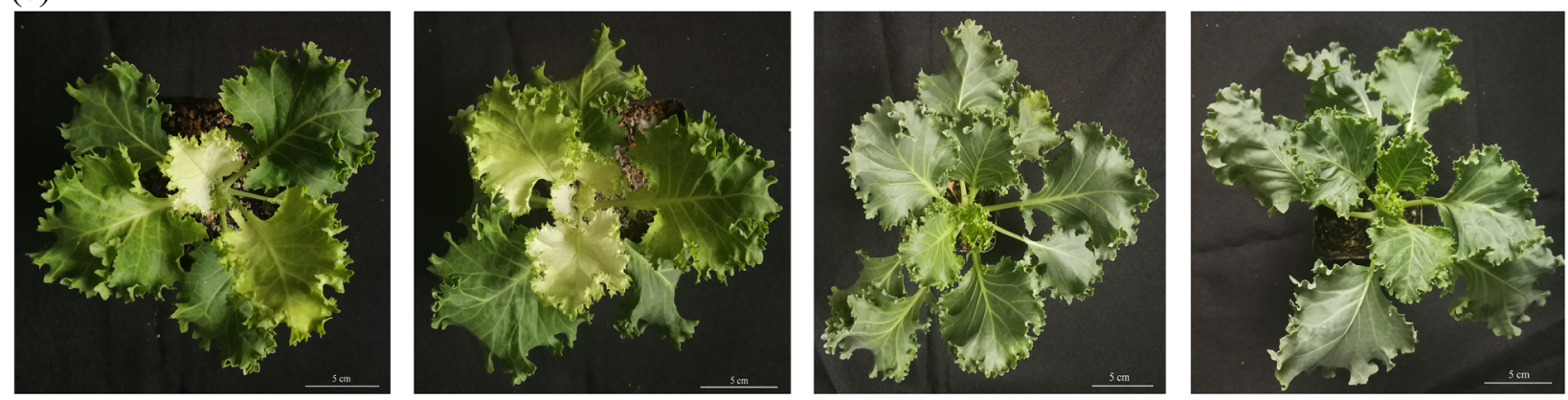

Fig. 6 Chlorophyll contents and phenotypes under different photoperiods and temperatures a Chlorophyll contents of WKO2 and green cabbage under different photoperiods (long-day and short-day) and temperatures $\left(24^{\circ} \mathrm{C}\right.$ and $10^{\circ} \mathrm{C}$ ) treatments. b Chlorophyll contents of WK02 under different temperature treatments. c The phenotypes of WKO2 under different temperature treatments including $4{ }^{\circ} \mathrm{C}, 10^{\circ} \mathrm{C}, 16^{\circ} \mathrm{C}$ and $24^{\circ} \mathrm{C}$ from left to right. LD, long-day photoperiod; SD, short-day photoperiod; WK, WKO2; GC, green cabbage; OL, outer leaves; IL, inner leaves. ${ }^{* * *}$ means $P<$ 0.001 by student's $t$ test. Bars $=5 \mathrm{~cm}$ 
Thus, the efficiency of map-based cloning was doubled by constructing suitable population.

The molecular mechanisms of albinism formation are complex. They are related to multiple biochemical processes, such as chlorophyll biosynthesis [25], carotenoid biosynthesis [26], and heme metabolism [27]. Low temperatures are usually considered a major abiotic stress that causes albinism in plants. Low-temperature-induced chlorosis/albinism, also called CTIC symptoms, has been reported in many higher plants, including Arabidopsis $[5]$ and rice [6, 7]. Generally, albino phenotypes are regarded as abnormal and negative traits in most plant breeding contexts [25]. However, the variegated and colourful leaves in ornamental kale are very popular among consumers because of their beautiful morphology [28]. Although a series of genes are related to chloroplast biogenesis at low temperatures, these mutants are usually controlled by recessive genes. In our study, two temperature-sensitive albino mutants, RK01 and WK02, were identified as showing a semi-dominant trait, rather than universal recessive inheritance. Using BSR-seq and linkage analysis, we narrowed the $A K$ gene to an approximate $60 \mathrm{~kb}$ region on chromosome C03. However, these genes in the target region had neither chlorophyll biosynthesis or breakdown genes, such as DVR [29], CHLG [30], CAO [31], nor any well-known genes that regulate chloroplast biogenesis, such as the PPR family [11]. These results suggest that the albinism in ornamental kale is probably controlled by some unknown mechanisms.

Plant genomic sequencing revealed that the cytochrome P450 gene family is one of the largest gene superfamilies in higher plants. The number of P450s is estimated to be approximately $1 \%$ of all annotated genes [32]. For instance, 246 and 356 P450s were identified in Arabidopsis and rice, respectively [33]. The functions of P450 genes in plants involve various biochemical reactions and biosynthesis processes, such as those related to sterols [34], plant hormones [32], defence compounds [35] and leaf development [36]. Based on the promoter variations and differentially expressed genes, Bol015404, encoding a cytochrome $\mathrm{P} 450$ protein, was selected as the most likely candidate gene for $A K$. In this study, Bol015404 was an uncharacterized gene belonging to the CYP708A subfamily. The expression of Bol015404 was induced by a low temperature of $10^{\circ} \mathrm{C}$ in the newly grown area. Therefore, upregulated expressions may lead to the development of albinism in the inner leaves of ornamental kale. Interestingly, CYP708 genes are absent in rice but present in Arabidopsis, suggesting differences between monocots and dicots in the corresponding metabolites [33]. In the CYP708A subfamily, CYP708A2 has been characterized as being involved in triterpene synthesis by operon-like clusters in Arabidopsis thaliana
[37]. However, the functions of other members of the CYP708A subfamily have not been validated in plants. Studies of the molecular mechanism of albinism in ornamental kale will broaden our knowledge of chloroplast development and biogenesis.

\section{Conclusions}

Two albino mutants with semi-dominant inheritance displaying CTIC symptoms were discovered in ornamental kale. We identified the target region harbouring the candidate gene for albinism using the BSR-seq method. The $A K$ gene was fine-mapped to a narrow region of 60 $\mathrm{kb}$, with a genetic distance of $0.33 \mathrm{cM}$. Thirteen genes were predicted in the mapping region, and the cytochrome P450 gene Bol015404 was selected as the most likely candidate gene for $A K$ based on its differential expression and promoter variations. The effects of temperature and light treatments revealed that the low temperatures, rather than the photoperiod, were the key factor for inducing albinism in ornamental kale. Additionally, the critical temperature for the albinism of ornamental kale was determined between $10^{\circ} \mathrm{C}$ and $16^{\circ} \mathrm{C}$ by the gradient test. The present study provided a novel type of albinism in higher plants, it also laid a foundation for understanding the genetic control of this trait in ornamental kale; a candidate gene for $A K$ was identified.

\section{Methods}

\section{Plant materials and phenotypes}

Two commercial varieties of ornamental kale (B. oleracea var. acephala) with albino phenotypes, Red Kamome and White Kamome (TAKII SEED, Japan), were used in this study. Two other materials, the green cabbage "HGDH" (B. oleracea var. capitata) and the curly kale "Zhou Ye Yu Yi" (Dongsheng Seeds, China), were utilized as normal parents for population construction. Green cabbage "HGDH" is a double haploid (DH) line, that was kindly provided by Professor Taotao Wang from Huazhong Agricultural University. All commercial materials were self-pollinated over three generations, confirming that the phenotypes were stable and consistent, especially those for leaf colour. Three parental lines derived from Red Kamome, White Kamome and "Zhou $\mathrm{Ye} \mathrm{Yu} \mathrm{Yi}^{\prime}$ by self-pollinated were named as RK01, WK02 and CK04, respectively. A backcross segregating population was generated by $\mathrm{RK} 01$ and green cabbage "HGDH" in our previous study [17]. The $\mathrm{BC}_{1}$ population can be used to elucidate the genetic mechanism of anthocyanin accumulation and albinism in ornamental kale, because two traits are independently controlled by two loci (Table S1). A $\mathrm{BC}_{1} \mathrm{~F}_{2}$ population was selected to further verify the genetic relationship of albino trait in ornamental kale. Additionally, an $\mathrm{F}_{2}$ population was generated by WKO2 and CK04 to analyse the genetic 
relationship of albino traits between Red Kamome and White Kamome. In late August or early September, all segregating populations were planted at the "Huangtupo" base at Huazhong Agricultural University (Wuhan, China). Albino phenotypes were first observed in late October and early November. The average high temperature and low temperature were $30^{\circ} \mathrm{C}$ and $20^{\circ} \mathrm{C}$ in September 2014. However, the average high temperature and low temperature decreased to $14{ }^{\circ} \mathrm{C}$ and $7{ }^{\circ} \mathrm{C}$ in November 2014, respectively (information derived from www.tianqi.com). These phenotypes were identified twice at four-month-old plants through visual observation. Four temperature and photoperiod treatmeats were performed in an artificial climate chamber. The long-day (LD) and short-day (SD) photoperiods were implemented under $16 \mathrm{~h} / 8 \mathrm{~h}$ (light/dark) and $8 \mathrm{~h} /$ $16 \mathrm{~h}$ (light/dark), respectively. Fluorescent lamps were used as the light source for plant growth, and the light density was approximately $280 \mu \mathrm{moles} / \mathrm{m}^{2} / \mathrm{s}$. The relative humidity (RH) was set at $75 \%$. To explore the critical temperature, a temperature gradient experiment was accomplished under the LD photoperiod with different temperatures, namely, $4^{\circ} \mathrm{C}, 10^{\circ} \mathrm{C}, 16^{\circ} \mathrm{C}$ and $24^{\circ} \mathrm{C}$. These above treatments were performed on three-week-old plants, and their phenotypes were identified after three weeks.

\section{Chlorophyll measurement}

Fresh leaves $(0.2 \mathrm{~g})$ were snipped into small pieces excluding vein and petiole. Chlorophyll was extracted with $10 \mathrm{~mL}$ ethanol solution $(96 \%, \mathrm{v} / \mathrm{v})$. The concentration of chlorophyll was measured at $649 \mathrm{~nm}$ and $665 \mathrm{~nm}$ [38]. In this study, chlorophyll contents of the parental lines were detected using four-month-old plants, and other treatment materials were measured at corresponding growth stages. All measurements were performed with four biological replications and three technical replications.

\section{Microscopy analysis of chloroplast}

Transmission electron microscopy was ultilized to observe the ultrastructure of chloroplasts. Samples were prepared following Cao's method [39]. To further observe the chloroplasts, protoplasts were isolated according to the procedure of Yoo et al. [40]. The protoplasts were recorded with inverted microscope (Olympus $1 \times 71$, Japan).

\section{BSR-seq analysis}

Bulked segregant analysis in combination with RNA-seq (BSR-seq) [18] were employed to identify the albino trait. For BSR-seq, two pools, the albino pool (A-pool, mutant pool) and the normal pool (N-pool, wild pool), were constructed by mixing an equal amount of tissues from 50 individuals of albino leaves and 50 individuals of normal leaves in the $\mathrm{BC}_{1}$ population, respectively. Total RNA was extracted from the two pools to accomplish the RNA sequencing using RNAiso plus kit method (Takara, Japan).

Pair-end (125 bp) libraries with insert sizes of approximate $350 \mathrm{bp}$ were prepared for sequencing on the Illumina Hiseq ${ }^{\mathrm{mi}} 2500$ platform. Approximate 5 GB clean data were generated by RNA-seq for each pool (NCBI Submission Archive, PRJNA580294). The alignments of paired-end reads were processed by the Hisat2 program [41] against reference genome 02-12 [42], and SNP callings were performed using SAMtools [43]. SNPindex and $\Delta$ (SNP-index) were computed to identify the candidate regions for the albino trait. The key parameter of the $\Delta$ (SNP-index) was calculated by subtracting the SNP-index of the N-pool from the SNP-index of the Apool. Confidence intervals (CIs) of 95 and $99 \%$ were computed for $\Delta$ (SNP-index) as described in a previous study [22]. The differentially expressed genes were analysed by FPKM (fragments per kilobase of transcript per million read pairs) values using the StringTie program [44].

\section{Molecular marker development and genetic mapping}

Several types of molecular markers were used in the $A K$ mapping (Table S2), including CAPS, Presence/absence, and SNP. Sequence specific primers for these markers were designed by using Primer 3.0 (http://primer3.ut.ee/) according to reference genome 02-12 [42]. A total of 93 randomly selected progenies in the $\mathrm{BC}_{1}$ population were used for preliminary mapping. The $A K$ gene was finemapped with 603 progenies of the $\mathrm{BC}_{1}$ population. Genotyping data of the $\mathrm{BC}_{1}$ individuals were utilized for linkage analysis by JoinMap4 program [45].

\section{Candidate gene prediction}

The differentially expressed genes were identified by StingTie [44] to get more information for the candidate gene selection. The genomic sequences in the target region were extracted from reference genome 02-12, and they were further predicted the Open Reading Frames (ORFs) by FGENESH (http://www.softberry.com/) and GENESCAN (http://genes.mit.edu/). The function annotations of these genes were retrieved from Blast2Go software (https://www.blast2go.com/) and TAIR website (https://www.arabidopsis.org/).

\section{Gene expression analysis}

Total RNA was isolated from fresh tissues with RNAiso plus reagent (Takara, Japan). The quality of RNA was measured by NanoDrop 2000 (ThermoFisher Scientific, USA). Furthermore, the value of $260 / 280>1.8$ was required for RNA samples in this study. The first-strand cDNA was 
synthesized by $2 \mu \mathrm{g}$ RNA using TransScript One-Step gDNA Removal and cDNA Synthesis SuperMix kit (TransScript, China). The synthesized cDNAs were diluted with $\mathrm{ddH}_{2} \mathrm{O}$ for RT-PCR. The quantitative RT-PCR was performed in QuantStudio 5 Real-Time PCR Systems (ThermoFisher Scientific, USA). The reaction volume was $10 \mu \mathrm{L}$ containing $5 \mu \mathrm{L}$ SYBR qPCR Master Mix (Vazyme, China), $0.3 \mu \mathrm{L}$ forward primer and reverse primer with $10 \mu \mathrm{M}$ concentration, $1 \mu \mathrm{L}$ cDNA template, and $3.4 \mu \mathrm{L} \mathrm{ddH}_{2} \mathrm{O}$. PCR amplification was processed by two-step cycling method of $95^{\circ} \mathrm{C}$ for $30 \mathrm{~s}$, and followed by 40 cycles of $95^{\circ} \mathrm{C}$ for $5 \mathrm{~s}$, and $60^{\circ} \mathrm{C}$ for 20 s. Melting curve was utilized to verify the specificity of primers. The expression levels of the candidate gene Bol015404 (qP450-F: GGGAAACATCCACAAGCA CA; qP450-R: TCTTTGGCCAGCCTTCAAAT) were detected with three biological replicates and three technical replications in several tissues including root, stem, inner leaves and outer leaves at $10^{\circ} \mathrm{C}$. The cabbage $\beta$-actin gene (AF044573) was used as the internal reference gene [46]. The relative expression levels were calculated with the formula $2^{-\Delta \Delta C q}$ [17]. Student's $t$ test was used to estimate significant differences among different samples.

\section{Supplementary information}

Supplementary information accompanies this paper at https://doi.org/10. $1186 /$ s 12870-020-02657-0.

Additional file 1: Table S1. The phenotypes of albino and anthocyanin traits in the BC1 population. Table S2. Molecular markers for mapping of $A K$ in C03. Figure S1. The phenotypes of albino and normal individuals in the $\mathrm{BC}_{1}$ population. Figure $\mathbf{S 2}$ The phenotypes of albino, slight albino and normal individuals in the $\mathrm{BC}_{1} \mathrm{~F}_{2}$ population. Figure $\mathbf{S 3}$. The alignment of coding sequences of Bol015404 alleles in WK02 (WK), RK01 (RK) and green cabbage (GC). Text S1 Genomic sequences of Bol015404 for RK01 (RK), WK01 (WK) and green cabbage(GC).

\section{Abbreviations}

CTIC: Cool-Temperature-Induced Chlorosis; BSR-seq: BSA + RNA-seq; SNP: Single Nucleotide Polymorphisim; CAPS: C1eaved Amplified PolymorphicSequences; LD: Long Day; SD: Short Day; Cls: Confidence Intervals

\section{Acknowledgments}

We thank Professor Robert Larkin and Professor Taotao Wang (Huazhong Agricultural University) for helping and advising in this project.

\section{Authors' contributions}

C.Y. finished the major experiments. L.Z. analysed the BSR-seg data. L.P. planted the population, detected the phenotypes and extracted DNAs. C.Y. wrote the manuscript with help from Z.Q. All authors have read and approved the manuscript.

\section{Funding}

This work was mainly supported by National Natural Science Foundation of China [31902058]; the Youth Science Fund of Hubei Academy of Agricultural Sciences [2020NKYJJ04]; and the China Agriculture Research System [CARS23--B-09]. All funders were not involved in the design of the study, data analysis, and writing.

\section{Availability of data and materials}

The datasets generated during the current study are available in the NCB (PRJNA580294).
Ethics approval and consent to participate

Not applicable.

\section{Consent for publication}

Not applicable.

\section{Competing interests}

The authors declare that they have no competing interests.

\section{Author details}

${ }^{1}$ Key Laboratory of Horticultural Plant Biology, Ministry of Education, College of Horticulture and Forestry Sciences, Huazhong Agricultural University, Wuhan 430070, People's Republic of China. ${ }^{2}$ Hubei Key Laboratory of Vegetable Germplasm Enhancement and Genetic Improvement, Institute of Economic Crops, Hubei Academy of Agricultural Sciences, Wuhan 430064, People's Republic of China.

Received: 19 May 2020 Accepted: 23 September 2020

Published online: 07 October 2020

\section{References}

1. Eckhardt U, Grimm B, Hörtensteiner S. Recent advances in chlorophyll biosynthesis and breakdown in higher plants. Plant Mol Bio. 2004;56(1):1-14

2. Stenbaek A, Jensen PE. Redox regulation of chlorophyll biosynthesis. Phytochemistry. 2010;71(2010):853-9.

3. Jensen PE, Leister D. Chloroplast evolution, structure and functions. F1000Prime Rep. 2014;6:40.

4. López-Juez E. Plastid biogenesis, between light and shadows. J Exp Bot. 2006:58(1):11-26.

5. Routaboul J-M, Fischer SF, Browse J. Trienoic fatty acids are required to maintain chloroplastfunction at low temperatures. Plant Physiol. 2000;124(4): 1697-705.

6. Yoshida R, Kanno A, Sato T, Kameya T. Cool-temperature-Induced Chlorosis in rice plants (I. relationship between the induction and a disturbance of etioplast development). Plant Physiol. 1996;110:997-1005.

7. Tao L, Liang KJ, Chen ZW, Duan YL, Wang JL, Ye N, Wu WR. Genetic analysis and gene mapping of cold-induced seedling chlorosis in rice. Hereditas. 2007;29:1121-5

8. Rodríguez VM, Velasco P, Garrido JL, Revilla P, Ordás A, Butrón A. Genetic regulation of cold-induced albinism in the maize inbred line A661. J Exp Bot. 2013;64(12):3657-67.

9. Dong Y, Dong W, Shi S, Jin Q. Identification and genetic analysis of a thermo-sensitive seedling-colour mutant in rice (Oryza sativa L.). Breed Sci. 2001:51(1):1-4.

10. Liu W, Fu Y, Hu G, Si H, Zhu L, Wu C, Sun Z. Identification and fine mapping of a thermo-sensitive chlorophyll deficient mutant in rice (Oryza sativa L.). Planta. 2007;226(3):785-95.

11. Su N, Hu ML, Wu DX, Wu FQ, Fei GL, Lan Y, Chen XL, Shu XL, Zhang X, Guo XP, Cheng ZJ, Lei CL, Qi CK, Jiang L, Wang H, Wan JM. Disruption of a rice pentatricopeptide repeat protein causes a seedling-specific albino phenotype and its utilization to enhance seed purity in hybrid rice production. Plant Physiol. 2012;159(1):227-38.

12. Wang $Y$, Zhang J, Shi $X$, Peng $Y$, Li $P$, Lin D, Dong $Y$, Teng S. Temperaturesensitive albino gene TCD5, encoding a monooxygenase, affects chloroplast development at low temperatures. J Exp Bot. 2016;67(17):5187-202

13. Yang MT, Chen SL, Lin CY, Chen YM. Chilling stress suppresses chloroplast development and nuclear gene expression in leaves of mung bean seedlings. Planta. 2005;221(3):374-85.

14. Linnaeus CS. Stockholm, Species Plantarum. Stockholm: Salvius; 1753.

15. Martin PG. Temperature-induced reversal of dominance of variegation in 'Ornamental kale'. Experientia. 1959; 15: 34-35.

16. Zhou S, Hu Z, Zhu M, Zhang B, Deng L, Pan Y, Chen G. Biochemical and molecular analysis of a temperature-sensitive albino mutant in kale named "white dove". Plant Growth Regul. 2013;71(3):281-94.

17. Yan C, An G, Zhu T, Zhang W, Zhang L, Peng L, Chen J, Kuang H. Independent activation of the BOMYB2 gene leading to purple traits in Brassica oleracea. Theor Appl Genet. 2019;132(4):895-906.

18. Liu S, Yeh CT, Tang HM, Nettleton D, Schnable PS. Gene mapping via bulked segregant RNA-Seq (BSR-Seq). PLoS One. 2012;7(5):e36406.

19. Takagi H, Abe A, Yoshida K, Kosugi S, Natsume S, Mitsuoka C, Uemura A Utsushi H, Tamiru M, Takuno S, Innan H, Cano LM, Kamoun S, Terauchi R. 
QTL-seq: rapid mapping of quantitative trait loci in rice by whole genome resequencing of DNA from two bulked populations. Plant J. 2013;74(1):17483.

20. Ren J, Liu Z, Du J, Fu W, Hou A, Feng H. Fine-mapping of a gene for the lobed leaf, BoLl, in ornamental kale (Brassica oleracea L. var. acephala). Mol Breeding. 2019;39(3):40

21. Feng $X, L i X$, Yang $X$, Zhu P. Fine mapping and identification of the leaf shape gene BoFL in ornamental kale. Theor Appl Genet. 2020;133(4):1303-12.

22. Tang $Q$, Tian M, An G, Zhang W, Chen J, Yan C. Rapid identification of the purple stem ( $P s)$ gene of Chinese kale (Brassica oleracea var. alboglabra) in a segregation distortion population by bulked segregant analysis and RNA sequencing. Mol Breeding. 2017:37(12):153.

23. Xu X, Luo W, Guo J, Chen H, Akram W, Xie D, Li G. Fine mapping and candidate gene analysis of the yellow petal gene ckpc in Chinese kale (Brassica oleracea L. var. alboglabra Bailey) by whole-genome resequencing. Mol Breeding. 2019;39:96.

24. Yan C, Huang Y, Liu Z, Guo F, Jiao Z, Yang W, Zhu F, Qiu Z. Rapid identification of yellow-flowered gene Bofc in cauliflower (Brassica oleracea var. botrytis) by bulked segregant analysis and whole-genome resequencing. Euphytica. 2020;216:26.

25. Ma Q, Li H, Zou Z, Arkorful E, Lv Q, Zhou Q, Chen X, Sun K, Li X. Transcriptomic analyses identify albino-associated genes of a novel albino tea germplasm 'Huabai 1'. Hortic Res. 2018;5(1):54.

26. Yuan H, Zhang J, Nageswaran D, Li L. Carotenoid metabolism and regulation in horticultural crops. Hortic Res. 2015;2:15036.

27. Zhang K, Mu Y, Li W, Shan X, Wang N, Feng H. Identification of two recessive etiolation genes (py1, py2) in pakchoi (Brassica rapa L. ssp. chinensis). BMC Plant Bio. 2020;20(1):68.

28. Schmidt S, Zietz M, Schreiner M, Rohn S, Kroh LW, Krumbein A. Genotypic and climatic influences on the concentration and composition of flavonoids in kale (Brassica oleracea var. sabellica). Food Chem. 2010;119(4):1293-9.

29. Wang P, Wan C, Xu Z, Wang P, Wang W, Sun C, Ma X, Xiao Y, Zhu J, Gao X. One divinyl reductase reduces the 8 -vinyl groups in various intermediates of chlorophyll biosynthesis in a given higher plant species, but the isozyme differs between species. Plant Physiol. 2013;161 (1):521-34.

30. Lin YP, Lee TY, Tanaka A, Charng YY. Analysis of an Arabidopsis heatsensitive mutant reveals that chlorophyll synthase is involved in reutilization of chlorophyllide during chlorophyll turnover. Plant J. 2015;80(1):14-26.

31. Sakuraba Y, Balazadeh S, Tanaka R, Mueller-Roeber B, Tanaka A. Overproduction of $\mathrm{Chl} b$ retards senescence through transcriptional reprogramming in Arabidopsis. Plant Cell Physiol. 2012;53(3):505-17.

32. Mizutani M, Ohta D. Diversification of P450 genes during land plant evolution. Annu Rev Plant Biol. 2010;61:291-315.

33. Nelson DR, Schuler MA, Paquette SM, Werck-Reichhart D, Bak S. Comparative genomics of rice and Arabidopsis. Analysis of 727 cytochrome P450 genes and pseudogenes from a monocot and a dicot. Plant Physiol. 2004:135(2):756-72.

34. Morikawa T, Mizutani M, Aoki N, Watanabe B, Ohta D. Cytochrome P450 CYP710A encodes the sterol C-22 Desaturase in Arabidopsis and tomato. Plant Cell. 2006;18(4):1008-22.

35. Jensen NB, Zagrobelny M, Hjernø K, Olsen CE, Houghton-Larsen J, Borch J, Møller BL, Bak S. Convergent evolution in biosynthesis of cyanogenic defence compounds in plants and insects. Nat Commun. 2011:2:273.

36. Anastasiou E, Kenz S, Gerstung M, MacLean D, Timmer J, Fleck C, Lenhard M. Control of plant organ size by KLUH/CYP78A5-dependent intercellular signaling. Dev Cell. 2007;13(6):843-56.

37. Field B, Osbourn AE. Metabolic diversification--independent assembly of operon-like gene clusters in different plants. Science. 2008;320(5875):543-7.

38. Lichtenthaler $\mathrm{H}$, Wellburn A. Determinations of total carotenoids and chlorophylls $a$ and $b$ of leaf extracts in different solvents. Biochem Soc Trans. 1983;11:591-2.

39. Cao H, Zhang J, Xu J, Ye J, Yun Z, Xu Q, Xu J, Deng X. Comprehending crystalline $\beta$-carotene accumulation by comparing engineered cell models and the natural carotenoid-rich system of citrus. J Exp Bot. 2012;63(12): 4403-17.

40. Yoo SD, Cho YH, Sheen J. Arabidopsis mesophyll protoplasts: a versatile cell system for transient gene expression analysis. Nat Protoc. 2007;2(7):1565-72.

41. Daehwan K, Ben L, Salzberg SL. HISAT: a fast spliced aligner with low memory requirements. Nat Methods. 2015;12(4):357-60.

42. Liu S, Liu Y, Yang X, Tong C, Edwards D, Parkin IA, Zhao M, Ma J, Yu J, Huang S, Wang X, Wang J, Lu K, Fang Z, Bancroft I, Yang TJ, Hu Q, Wang X,
Yue Z, Li H, Yang L, Wu J, Zhou Q, Wang W, King GJ, Pires JC, Lu C, Wu Z, Sampath P, Wang Z, Guo H, Pan S, Yang L, Min J, Zhang D, Jin D, Li W, Belcram H, Tu J, Guan M, Qi C, Du D, Li J, Jiang L, Batley J, Sharpe AG, Park BS, Ruperao P, Cheng F, Waminal NE, Huang Y, Dong C, Wang L, Li J, Hu Z, Zhuang M, Huang Y, Huang J, Shi J, Mei D, Liu J, Lee TH, Wang J, Jin H, Li Z, Li X, Zhang J, Xiao L, Zhou Y, Liu Z, Liu X, Qin R, Tang X, Liu W, Wang Y, Zhang Y, Lee J, Kim HH, Denoeud F, Xu X, Liang X, Hua W, Wang X, Wang J, Chalhoub B, Paterson AH. The Brassica oleracea genome reveals the asymmetrical evolution of polyploid genomes. Nat Commun. 2014;5:3930.

43. Li H, Handsaker B, Wysoker A, Fennell T, Ruan J, Homer N, Marth G, Abecasis $G$, Durbin R. The sequence alignment/map format and SAMtools. Bioinformatics. 2009;25(16):2078-9.

44. Pertea M, Kim D, Pertea GM, Leek JT, Salzberg SL. Transcript-level expression analysis of RNA-seq experiments with HISAT, StringTie and Ballgown. Nat Protoc. 2016;11(9):1650-67.

45. Van Ooijen JW. JoinMap4: Software for the calculation of genetic linkage maps in experimental populations. Wageningen: Kyazma BV; 2006.

46. Yuan Y, Chiu LW, Li L. Transcriptional regulation of anthocyanin biosynthesis in red cabbage. Planta. 2009;230(6):1141-53.

\section{Publisher's Note}

Springer Nature remains neutral with regard to jurisdictional claims in published maps and institutional affiliations.
Ready to submit your research? Choose BMC and benefit from:

- fast, convenient online submission

- thorough peer review by experienced researchers in your field

- rapid publication on acceptance

- support for research data, including large and complex data types

- gold Open Access which fosters wider collaboration and increased citations

- maximum visibility for your research: over $100 \mathrm{M}$ website views per year

At BMC, research is always in progress.

Learn more biomedcentral.com/submissions 